\title{
Diseño de un modelo para evaluar la accesibilidad web y validación sobre un ranking de las universidades españolas
}

\author{
Design of a model for assessing Web accessibility and validation on a raking of the Spanish universities
}

\section{Maypher RomÁn Durán (1), Jose Luis Bernier VilLAMOR (2), Gerardo FERnÁndez Rodríguez (2)}

(1) Universidad de Ciencias Informáticas (UCI), La Habana, Cuba, maypher@uci.cu

(2) Universidad de Granada, Granada, España, jbernier@ugr.es

\begin{abstract}
Resumen
Se presenta un estudio sobre los sitios Web de las universidades españolas, y el diseño de una medida cuantitativa para medir su nivel de accesibilidad, con objeto de establecer un ranking que permita evaluarlas y compararlas entre sí. En este estudio se han considerado 79 universidades españolas, cuyos sitios web institucionales se han analizado en profundidad midiendo diversos indicadores relacionados con la accesibilidad web, tales como la satisfacción de estándares y directrices de accesibilidad, la compatibilidad con distintos navegadores, errores de usabilidad, etc. A partir de los resultados obtenidos y con la métrica propuesta, se ha elaborado un ranking de universidades que pretende medir la calidad de sus sitios institucionales en cuanto a accesibilidad web. El objetivo del presente trabajo es presentar los resultados obtenidos a partir de dicho estudio.
\end{abstract}

Palabras clave: Accesibilidad Web. Ranking. Universidades. Métricas.

\section{Introduction}

El mundo web nació hace apenas unos 20 años, pero en estas dos décadas su crecimiento y poder de penetración en la sociedad ha sido impresionante. Cada vez hay una mayor dependencia del ciudadano respecto de la Web, no sólo para obtener información, sino también para acceder a cada vez más servicios (sanitarios, educativos, comerciales, etc.). Lamentablemente, en la sociedad existen personas que pueden tener dificultades para aprovechar todas estas ventajas.

Se estima que hay unos 650 millones de personas con discapacidad en el mundo. Según datos publicados por el Instituto Nacional de Estadísticas (INE), de un estudio realizado en 2008 , en España hay alrededor de 3,7 millones de personas que sufre algún tipo de discapacidad (INE, 2013). Se entiende por discapacidad una limitación que presente una persona para realizar determinas funciones, pudiendo ser esta

\begin{abstract}
This paper presents a study of the websites of the Spanish universities, and the design of a quantitative metric for measuring their level of accessibility, in order to establish a ranking that can be used for evaluate and compare them. This study considered the website of 79 Spanish universities; they have been analyzed by measuring some web accessibilityrelated indicators, such as accessibility and standards guidelines compliance, compatibility, usability errors, etc. With the obtained results and the proposed metric, we have developed a ranking of universities that measure their web accessibility quality. The main goal of this paper is presenting the results of this study.
\end{abstract}

Keywords: Web accessibility. Universities. Rankings. Metrics.

física-motora, visual, mental, auditiva, visceral o mixta (Colectivo, 2003, p. 87-9).

Las personas con discapacidad pueden encontrarse con serios problemas para acceder a los contenidos de una página web, entre los que se pueden citar: no ser capaz de ver, escuchar, seleccionar o comprender algunos tipos de información fácilmente o en absoluto; dificultad en la lectura o comprensión de un texto; no ser capaz de usar un teclado o un ratón; tener una pantalla que sólo presenta texto, una pantalla pequeña o una conexión lenta a Internet; no hablar o comprender con fluidez el idioma en que esté redactado el documento. Los desarrolladores de sitios web deben tener en cuenta estas diferentes situaciones si quieren evitar que estas personas se encuentren con problemas de acceso a los contenidos.

El Consorcio W3C (World Wide Web) ha trazado políticas para definir las pautas de accesibilidad de los contenidos en la Web, las llamadas WCAG (Web Content Accessibility Guidelines). 
(WCAG, 2008) Las mismas tratan los aspectos de accesibilidad y proporcionan soluciones de diseño accesibles. Siguiendo estas pautas, los desarrolladores de contenidos pueden crear páginas accesibles, desde un punto de vista técnico.

Un estudio del 2008 de las agencias de gobierno en 12 países, localizados en la Unión Europea, Asia y África, arrojó que la mayoría de los sitios Web no cumplían con los requisitos de prioridad 1 del estándar WCAG. Incluso en esos países que poseen leyes internas para asegurar la accesibilidad de sus sitios, pocos sitios cumplieron con lo establecido en el estándar (Kuzma, 2009). Otro estudio realizado en 2009 en el Reino Unido de los sitios Web de los Miembros del Parlamento también mostró que la mayoría no cumplía tampoco con las leyes establecida en el país para la compatibilización de los sitios públicos con los estándares de la W3C (Kuzma, 2010). Por otra parte la legislación española regula desde 2007 las tecnologías y estándares que deben contemplar las páginas web de los organismos públicos, estableciendo un conjunto de requisitos mínimos que éstas deben asegurar, y que, por tanto, es necesario conocer y cumplir. En concreto la ley 49/2007, de 26 de diciembre, establece sanciones que van desde los 301 euros hasta el millón de euros por el incumplimiento de las normativas de accesibilidad web.

A nivel internacional son muchos los rankings que han surgido para clasificar y ordenar las universidades de acuerdo a diferentes criterios (Hilera, 2013). Tienen dos propósitos fundamentales: orientar a los estudiantes en la elección de un centro de estudios y brindar información útil a los gestores de la universidad sobre sus fortalezas y debilidades. Sin embargo, estos rankings no evalúan la calidad del sitio web de las universidades. Para ordenar las universidades españolas se han propuesto otros, como por ejemplo el Ranking ISI desarrollado por especialistas de la Universidad de Granada (Herrera, 2011). La universidad pueden tener resultados excelentes en producción científica; sin embargo, su sitio web puede presentar problemas de accesibilidad para algunas personas.

Se tiene referencia en España de la propuesta de un ranking que tiene en cuenta los sitios Web de las Universidades: el Ranking Web (Webometrics) de Universidades (Webometrics, 2013). Este ranking mide la visibilidad y la actividad del sitio, es decir, la calidad de sus contenidos publicados, haciendo énfasis en el valor de la información y la utilidad de los servicios que ofrece. Tiene en cuenta además el tráfico del sitio, su presencia en internet, la cantidad de sitios que tienen enlace a los contenidos del sitio de la universidad desde diferentes dominios, y la presencia de repositorios institucionales para publicar los resultados como forma de transferencia de conocimientos científicos y culturales que ha generado la universidad, entre otros aspectos. Este ranking no evalúa el diseño del sitio, la usabilidad o el cumplimiento de estándares de accesibilidad. Otros estudios internacionales proponen la construcción de un ranking de sitios web de universidades a partir del análisis de la accesibilidad de dichos sitios. Estos trabajos evalúan solamente la página principal (Rivera, 2009) o una muestra de tres páginas relevantes de cada sitio web (Hilera, 2013), y no reflejan un análisis global de todas las páginas del sitio o de una muestra significativa del mismo.

El objetivo de este trabajo es diseñar un ranking de las universidades españolas, a partir del nivel de accesibilidad de su sitio web en Internet. Se describe la metodología y métricas propuestas para su elaboración.

\section{Metodología}

Identificar los problemas de accesibilidad presentes en un sitio web se convierte en un análisis que, si bien no es un proceso del todo complejo, sí demanda un gran consumo de tiempo y conocimiento por parte del especialista que realiza dicho estudio; el cual debe conocer cada una de las pautas asociadas a la accesibilidad Web. Existen hoy herramientas que pueden ser utilizadas para evaluar el cumplimiento de estas pautas en los contenidos de una página (conformidad con las normas WCAG), conocidos como validadores. Tal es el caso de la herramienta SortSite, que permite hacer un análisis detallado de un sitio web completo o una parte del mismo, validando un gran número de aspectos (SortSite, 2013).

Para realizar el estudio sobre la accesibilidad de los sitios Web de las 79 universidades españolas (Ministerio, 2012), se ha diseñado una estrategia que permite organizar todo el proceso. El nivel de profundidad usado para el análisis fue 3. Es decir, a partir de la página principal del sitio (portada) fueron analizadas de forma exhaustiva todas aquellas páginas y archivos que estaban enlazados desde la misma, hasta un nivel de profundidad de tres clics. A cada página considerada se le aplicó una batería de test para analizar numerosas cuestiones relacionadas con la accesibilidad y cumplimiento de estándares web, en total se consideraron 35 tests de diversa índole sobre cada página analizada en cada sitio web. 


\subsection{Aspectos analizados}

En el estudio se realizaron de forma exhaustiva una serie de test de validación en cada sitio web, que se agruparon en las siguientes categorías generales:

- Accesibilidad: Se verifica el cumplimiento de las directrices de accesibilidad, los formatos de archivo y se identifican los errores de prioridad A, AA y AAA tanto para WCAG 1.0 como WCAG 2.0.

- Estándares Web: Se verifica el cumplimiento de los estándares establecidos por la W3C: HTML/XHTML y CSS.

- Búsqueda: Se comprueban las guías de optimización establecidas por los principales motores de búsqueda: Google, Bing y Yahoo, así como buenas prácticas recomendadas por expertos en posicionamiento web.

- Usabilidad: Se verifican las directrices de Usability.gov de US, las de W3C y las recomendadas por Wikipedia. Se identifican errores asociados con imágenes corruptas, páginas que tardan en cargarse, errores ortográficos, texto excesivamente pequeño para lectura, etc.

- Calidad: Se detectan los enlaces rotos, errores de scripts y de configuración, archivos que presentan errores de formato y errores de sintaxis.

Como herramienta de validación se ha utilizado SortSite Professional en su versión 5.1, la cual permite realizar un análisis automático de la mayor parte de los aspectos a medir.

\subsection{Indicadores de error}

Los indicadores de errores a medir son esenciales a la hora de construir el ranking. A partir de los indicadores medidos se diseñó un valor denominado WAU-index (University Web Accessibility index). El mismo se puede definir como un valor cuantitativo que permite ordenar un conjunto de universidades en función de la accesibilidad de su sitio web y comparar su calidad. Para el cálculo de éste valor final fueron definidos 5 indicadores a partir de los aspectos evaluados descritos en la sección 2.1 .

- Indicador de accesibilidad $\left(I_{A c c}\right)$.

- Indicador de estándares $\left(I_{E s t}\right)$.

- Indicador de búsqueda ( $\left.I_{\text {Bus }}\right)$.

- Indicador de usabilidad (IUsa).

- Indicador de calidad $\left(I_{C a l}\right)$.
Cada indicador $I_{x}$ mide el nivel de error que presenta un sitio web en la categoría $x$ considerada. Un valor menor de $I_{x}$ significa que el sitio web presenta menos errores en la categoría $x$.

Para el cálculo de cada uno de los 5 indicadores anteriores se usa la fórmula siguiente:

$$
I_{x}=P E(x) \quad \sum_{i}\left(E_{i}(x) \quad W_{i}\right)
$$

Donde:

- $I_{x}$ : Se refiere a cualquiera de los coeficientes citados, siendo $x$ una de las categorías analizadas (accesibilidad, estándares, búsqueda, usabilidad y calidad).

- $P E(x)$ : Porcentaje de páginas que presentan errores en la categoría $x$, respecto del total de páginas analizadas en el sitio web. Este valor se expresa en tanto por uno, con objeto de trabajar con valores normalizados.

- $E_{i}(x)$ : Tanto por uno de errores de tipo $i$ (críticos, importantes, regulares o menores) con respecto al total de errores encontrados en la categoría $x$.

- $W_{i}$ : peso asignado a cada error tipo $i$ para jerarquizar su importancia. Se han tomado como valores $0.4,0.3,0.2$ y 0.1 para ponderar la gravedad del error.

Los valores $E_{i}$ y $W i$ se han definido para distinguir la gravedad de los errores detectados en cada categoría. Los errores detectados se han clasificado en 4 categorías atendiendo a su gravedad: críticos, importantes, regulares y menores. Por ejemplo, en el caso del indicador de accesibilidad $\left(I_{A C C}\right)$ los errores críticos se asocian al incumplimiento del nivel de prioridad 1 , los importantes se relacionan con el nivel de prioridad 2 y el resto con el nivel 3. De igual forma, se distingue la gravedad del error en el resto de categorías para calcular el indicador correspondiente.

La Tabla I muestra los resultados obtenidos en la categoría de accesibilidad de tres sitios Web analizados. Por ejemplo, para calcular el coeficiente de accesibilidad del sitio $A$, se realizaría la siguiente operación:

$$
\begin{aligned}
I_{\text {AcC }}= & 0.1673 *((14 / 26) * 0.4+(9 / 26) * 0.3+ \\
& (3 / 26) * 0.2+(0 / 26) * 0.1)=0.0572
\end{aligned}
$$

Como se puede apreciar, además de distinguir la importancia que tienen los diferentes tipos de errores, es necesario tener en cuenta el porcentaje de páginas con errores asociada a cada coeficiente. Es decir, no sólo es relevante la tipología de errores y su ocurrencia, sino también el total de páginas donde éstos fueron de- 
tectados. Hay que tener en cuenta que, a igualdad del número de errores, no se pueden valorar de la misma forma un sitio web con solo 30 páginas que otro con 300; de ahí que se use el valor $P E_{X}$ para el cálculo del indicador. Este valor tiene como objetivo equilibrar los indicadores considerando el volumen de documentos (páginas, imágenes, archivos, etc.) que componen el sitio web analizado.

Como ejemplo de lo expuesto, puede observarse a partir de la Tabla I que, a pesar de que el sitio $C$ tuvo más errores Críticos que el sitio $B$ (41 y 15 respectivamente), su indicador de accesibilidad presenta un valor menor. Esto se debe a que el número de páginas con errores de accesibilidad del sitio $C(19 \%)$ fue bastante menor que el del sitio B (28\%).

\begin{tabular}{lccc}
\hline Medidas & Sitio & Sitio Web & Sitio Web \\
& Web $A$ & $B$ & $C$ \\
\hline \# págs analizadas & 496 & 272 & 569 \\
\hline $\begin{array}{l}\text { \# págs con error de } \\
\text { accesibilidad }\end{array}$ & 83 & 77 & 107 \\
\hline$P E(A c c)$ & 0.17 & 0.28 & 0.19 \\
\hline \# Críticos $\left(E_{C}(\right.$ Acc $\left.)\right)$ & 14 & 15 & 41 \\
\hline $\begin{array}{l}\text { \# Importantes } \\
\left(E_{l}(\text { Acc })\right)\end{array}$ & 9 & 8 & 15 \\
\hline \# Regulares $\left(E_{R}(A c c)\right)$ & 3 & 3 & 3 \\
\hline \# Menores $\left(E_{M}(A c c)\right)$ & 0 & 0 & 0 \\
\hline$W_{\text {Criticos }}$ & 0.4 & 0.4 & 0.4 \\
\hline$W_{\text {Importantes }}$ & 0.3 & 0.3 & 0.3 \\
\hline$W_{\text {Regulares }}$ & 0.2 & 0.2 & 0.2 \\
\hline$W_{\text {Menores }}$ & 0.1 & 0.1 & 0.1 \\
\hline Valor de $\mathrm{I}_{\text {Acc }}$ & 0.0572 & 0.09792 & 0.06852 \\
\hline
\end{tabular}

Tabla I. Medidas del análisis de la categoría Accesibilidad de varios sitios web

\subsection{Coeficientes de calidad}

Una vez calculados los cinco indicadores de error para cada uno de los sitios web analizados, se procedió a calcular sus coeficientes de calidad asociados.

Para obtener estos coeficientes, en primer lugar se procedió a normalizar cada indicador. Para ello, por cada sitio web considerado, se dividió su indicador entre el valor máximo encontrado en la categoría correspondiente. El objetivo de esta operación es fijar los valores de los indicadores entre los valores máximo y mínimo del conjunto analizado.
En un segundo paso, se procedió a restar 1 de cada indicador para obtener los coeficientes. El objetivo fue obtener una medida positiva en la que un valor mayor represente mayor calidad. Tal y como se ha definido previamente, el valor máximo de un indicador implica un número mayor de errores en la categoría considerada. Al dividir por dicho máximo, se obtienen valores cercanos al 1 en aquellos sitios con más errores y cercanos al 0 en caso contrario. Al restar de 1 se invierte el valor conceptual de la medida, de forma que un valor cercano a 1 implica mayor calidad.

Como ejemplo de cálculo, la Tabla II muestra el valor de los coeficientes finales, tal y como se ha indicado, de cuatro de los sitios web analizados. $I_{A c c}$ es el indicador de la categoría Accesibilidad medido, $I_{M}(A c c)$ es el indicador máximo encontrado en dicha categoría (se ha resaltado en negrita en la tabla). $C_{A c c}$ es el valor final del coeficiente que evalúa la calidad del sitio web con respecto a la categoría Accesibilidad. Según el ejemplo considerado, el sitio $B$ tiene mejor puntuación en la categoría de Accesibilidad.

\begin{tabular}{lccc}
\hline Sitio Web & $I_{A c c}$ & $I_{A c d} I_{M}(A c c)$ & $C_{A c c}$ \\
\hline Sitio A & 0,178 & 0,446 & 0,554 \\
\hline Sitio B & 0,029 & 0,072 & 0,928 \\
\hline Sitio C & 0,163 & 0,406 & 0,594 \\
\hline Sitio D & $\mathbf{0 , 4 0 0}$ & 1 & 0 \\
\hline
\end{tabular}

Tabla II. Obtención de los coeficientes de accesibilidad a partir de sus indicadores

El mismo procedimiento se llevó a cabo con el resto de indicadores para obtener los coeficientes de calidad de cada categoría.

\subsection{Medida global de calidad}

El indicador de calidad web WAU-index se ha definido a partir de los coeficientes de calidad anteriormente expuestos según la siguiente expresión:

$$
W A U-\text { Index }=\sum_{x} C_{x} W_{x}
$$

Donde:

- $C_{X}$ : Se refiere a cada uno de los coeficientes propuestos, correspondientes a las cinco categorías analizadas (accesibilidad, estándares, búsqueda, usabilidad y calidad).

- $W(\mathrm{x})$ : Se refiere al peso asignado a cada coeficiente, y en consecuencia, a cada categoría analizada. 
La expresión utilizada calcula la suma ponderada de los coeficientes de calidad obtenidos. Los valores considerados para ponderar cada categoría fueron propuestos de forma heurística, desde el punto de vista de la accesibilidad Web que tiene cada aspecto evaluado. El valor utilizado para estos pesos se muestra en la Tabla III.

\begin{tabular}{lc}
\hline Pesos & Valor \\
\hline$W_{\text {Acc }}$ & 0.3 \\
\hline$W_{\text {Est }}$ & 0.3 \\
\hline$W_{\text {Bus }}$ & 0.1 \\
\hline$W_{\text {Usa }}$ & 0.1 \\
\hline$W_{\text {Cal }}$ & 0.2 \\
\hline
\end{tabular}

Tabla III. Valores usados para la ponderación de cada coeficiente evaluado

\begin{tabular}{lcc}
\hline Coeficientes & Valor & Peso \\
\hline$C_{A c c}$ & 0,857 & 0.3 \\
\hline$C_{\text {Est }}$ & 0,907 & 0.3 \\
\hline$C_{\text {Bus }}$ & 0,818 & 0.1 \\
\hline$C_{\text {Usa }}$ & 0,889 & 0.1 \\
\hline$C_{\text {Cal }}$ & 0,903 & 0.2 \\
\hline
\end{tabular}

Tabla IV. Cálculo del indicador WAU-index

Puede observarse que la suma de los mismos es igual a 1. Dicho peso se propuso básicamen- te atendiendo al cumplimiento de las normas normativa UNE 13980 del 2012 (UNE, 2012), donde se indica que de cara a la accesibilidad debe satisfacerse: WCAG hasta prioridad 2 y el respeto de estándares web. La Tabla IV muestra los valores de los coeficientes de calidad para las cinco categorías consideradas en este estudio para un sitio web determinado.

Según los datos de la Tabla IV, el coeficiente WAU-index se calcularía de la siguiente forma:

$$
\begin{aligned}
\text { WAU-index }= & 0,857 * 0.3+0,907 * 0.3 \\
& +0,818 * 0.1+0,889 * 0.1 \\
& +0,903 * 0.2=0,881
\end{aligned}
$$

Hay que resaltar que el uso de las expresiones propuestas, y la metodología detallada en este trabajo han sido una primera aproximación, pero no se descarta estudiar otras alternativas, tales como el uso de la media geométrica como se realiza en Herrera (2011), o considerar otras heurísticas y técnicas para asignar valores a los pesos considerados.

\section{Resultados y discusión}

Fueron analizados los sitios web principales de las 79 universidades españolas. La Tabla V resume las 25 universidades que presentaron un mejor sitio web, ordenadas en orden decreciente atendiendo al valor del índice WAU calculado. Se muestran los valores de sus coeficientes de calidad y del indicador final WAU.

\begin{tabular}{llllllll}
\hline Posición & Índice WAU & Universidad & $C_{A c c}$ & $C_{\text {Est }}$ & $C_{\text {Bus }}$ & $C_{\text {Usa }}$ & $C_{\text {Cal }}$ \\
\hline 1 & 0,949 & Universidad de Valladolid & 0,945 & 0,914 & 0,964 & 0,975 & 0,948 \\
\hline 2 & 0,915 & Universidad de Almería & 0,916 & 0,943 & 0,905 & 0,910 & 0,904 \\
\hline 3 & 0,893 & Universidad de Burgos & 0,800 & 0,892 & 0,896 & 0,908 & 0,968 \\
\hline 4 & 0,874 & Universidad de Granada & 0,856 & 0,906 & 0,817 & 0,888 & 0,902 \\
\hline 5 & 0,885 & Universitat Jaume I & 0,797 & 0,957 & 0,946 & 0,908 & 0,918 \\
\hline 6 & 0,894 & Universidad Católica de San Antonio & 0,927 & 0,785 & 0,970 & 0,988 & 0,800 \\
\hline 7 & 0,874 & Universidad San Jorge & 0,771 & 0,823 & 0,913 & 0,894 & 0,971 \\
\hline 8 & 0,873 & Universitat Politécnica de Catalunya & 0,857 & 0,753 & 0,914 & 0,949 & 0,912 \\
\hline 9 & 0,858 & Universidad de Oviedo & 0,950 & 0,927 & 0,980 & 0,975 & 0,460 \\
\hline 10 & 0,856 & Universidad de Salamanca & 0,752 & 0,551 & 0,852 & 0,840 & 0,831 \\
\hline 11 & 0,852 & Universidade da Coruña & 0,747 & 0,799 & 0,886 & 0,881 & 0,937 \\
\hline 12 & 0,851 & Universitat Pompeu Fabra & 0,826 & 0,716 & 0,931 & 0,922 & 0,864 \\
\hline 13 & 0,851 & Universidad de La Laguna & 0,768 & 0,685 & 0,935 & 0,900 & 0,965 \\
\hline 14 & 0,833 & Universidad de Alicante & 0,802 & 0,718 & 0,902 & 0,844 & 0,901 \\
\hline 15 & 0,833 & Universitat de València & 0,753 & 0,737 & 0,885 & 0,895 & 0,900 \\
\hline
\end{tabular}


54

\begin{tabular}{llllllll}
\hline 16 & 0,832 & Universidad de Las Palmas de Gran Canaria & 0,791 & 0,675 & 0,859 & 0,883 & 0,952 \\
\hline 17 & 0,817 & Universidad de Vigo & 0,756 & 0,769 & 0,840 & 0,850 & 0,868 \\
\hline 18 & 0,813 & Mondragon Unibertsitatea & 0,828 & 0,638 & 0,919 & 0,928 & 0,851 \\
\hline 19 & 0,808 & Universitat Rovira i Virgili & 0,697 & 0,766 & 0,830 & 0,840 & 0,909 \\
\hline 20 & 0,807 & Universidad Complutense de Madrid & 0,825 & 0,623 & 0,921 & 0,886 & 0,839 \\
\hline 21 & 0,804 & Universidad Nacional de Educación a Distancia & 0,842 & 0,607 & 0,940 & 0,957 & 0,777 \\
\hline 22 & 0,804 & Universidad de Cantabria & 0,754 & 0,625 & 0,908 & 0,897 & 0,908 \\
\hline 23 & 0,803 & Universidad de Cádiz & 0,733 & 0,762 & 0,909 & 0,891 & 0,729 \\
\hline 24 & 0,802 & Universitat de Barcelona & 0,753 & 0,626 & 0,897 & 0,907 & 0,889 \\
\hline 25 & 0,800 & Universidad Internacional de Andalucía & 0,715 & 0,656 & 0,854 & 0,867 & 0,910 \\
\hline
\end{tabular}

Tabla V. Ranking de Accesibilidad Web de universidades españolas

La siguiente figura muestra la cantidad de universidades cuyos valores del índice WAU se encuentran comprendidos en distintos rangos:

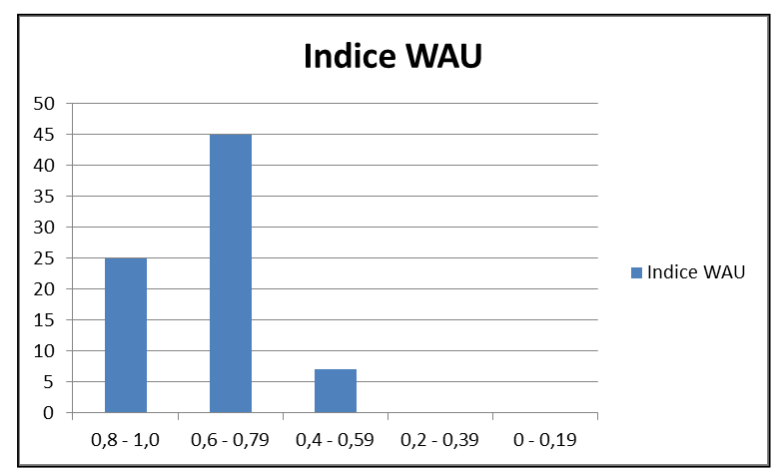

Figura 1. Cantidad de universidades españolas agrupadas por rangos del valor del índice WAU

\begin{tabular}{lll}
\hline Posición & Comunidad & WAU-index \\
\hline 1 & Principado de Asturias & 0,851 \\
\hline 2 & Castilla y León & 0,752 \\
\hline 3 & $\begin{array}{l}\text { Comunidad Foral de } \\
\text { Navarra }\end{array}$ & 0,728 \\
\hline 4 & Galicia & 0,728 \\
\hline 5 & Canarias & 0,719 \\
\hline 6 & Catalunya & 0,711 \\
\hline 7 & Extremadura & 0,709 \\
\hline 8 & Aragón & 0,694 \\
\hline 9 & Región de Murcia & 0,677 \\
\hline 10 & Comunidad de Madrid & 0,669 \\
\hline 12 & Illes Balears & 0,659 \\
\hline 13 & Cantabria & 0,658 \\
\hline 14 & Comunidad Valenciana & 0,651 \\
\hline 15 & Andalucía & 0,639 \\
\hline 16 & País Vasco & 0,632 \\
\hline 17 & La Rioja & 0,585 \\
\hline & Castilla La Mancha & 0,544 \\
\hline
\end{tabular}

Tabla VI. Ranking de Accesibilidad Web de universidades por comunidad autónoma.
Siguiendo la misma metodología propuesta, se propuso un ranking de accesibilidad de las comunidades autónomas, calculando el valor medio del índice WAU de sus universidades:

La Tabla VI muestra el resultado del índice WAU medio por comunidades autónomas.

\section{Conclusiones}

Se ha presentado una metodología para el diseño de un ranking de accesibilidad web. Fueron identificados cinco indicadores que se utilizan para evaluar cada una de las categorías seleccionadas en el estudio: accesibilidad, estándares, búsqueda, usabilidad y calidad. Para el cálculo de cada uno de estos indicadores se consideraron cuatro tipos de errores posibles: críticos, importantes, regulares y menores, de acuerdo a su nivel de importancia.

Se han analizado los sitios web principales de las 79 universidades españolas. El proceso de validación de los sitios web se realizó de forma automática con el uso de la herramienta SortSite Professional en su versión 5.1, con la cual se han realizado una batería de 35 test de validación sobre cada página analizada. En cada sitio web se han analizado de forma exhaustiva todas aquellas páginas que distaban hasta tres clics de profundidad respecto de la página de inicio.

Como medida final de ordenación de las universidades se diseñó una métrica denominada WAU-index (University Web Accessibility index). El índice WAU se puede tomar como un indicador cuantitativo que permite comparar y ordenar un conjunto de sitios web en función de su accesibilidad. Como ejemplo de uso se han analizado los sitios web de 79 universidades españolas y se ha establecido un ranking con los resultados obtenidos. 
Como trabajo futuro, se pretende estudiar otras posibles métricas y metodologías de cálculo para el índice propuesto, así como realizar un estudio de correlación de los indicadores usados. Se pretende continuar analizando la evolución de los sitios web de cada universidad estudiada, con el fin de elaborar versiones actualizadas del ranking con cierta periodicidad y hacer públicos los resultados e informes. También se pretende aplicar esta metodología a otras instituciones, y tratar de concluir nuevas relaciones y conocimientos a partir de los análisis de resultados.

\section{Referencias}

Colectivo de autores (2003). Por la Vida: Estudio Clínico Genético de las personas con retraso mental. Definiciones de discapacidad y criterios de inclusión y exclusión en el estudio. La Habana, Cuba: Casa Editora Abril, 2003. 87-90.

Herrera, Francisco, Torres-Salinas, Daniel; Moreno-Torres, Jose G.; Delgado-Lopez-Cozar, Emilio (2011). A methodology for Institution-Field ranking based on a bidimensional analysis: the IFQ2A index. // Scientometrics. 88, 771-786. DOI 10.1007/s11192-011-0418-6, 2011.

Hilera, José R.; Fernández, Luis; Suárez, Esther; Vilar, Elena T. (2013). Evaluación de la accesibilidad de páginas web de universidades españolas y extranjeras incluidas en rankings universitarios internacionales. // Revista Española de Documentación Científica. 36:1. ISSN-L: 0210-0614.
Instituto Nacional de Estadística (INE). http://www.ine.es/ (Marzo, 2013).

Kuzma, Joanne M. (2010). Accessibility design issues with UK e-government sites. // Government Information Quarterly. (2010).

Kuzma, Joanne M., Yen, D., Oestreicher, K. (2009). Global E-Government web accessibility: An empirical examination of EU, Asian and African sites. // Second International Conference on Information and Communication Technologies and Accessibility. Hammamet, Tunisia: Mayo 2009.

Ministerio de Educación, Cultura y Deporte (2012). Datos y cifras del sistema universitario español: Curso 20122013. Madrid: Secretaría General Técnica, Subdirección general de documentación y publicaciones; Solana e hijos A.G., S.A.U. España.

Ranking Web de Universidades Webometrics (2013). http://www.webometrics.info/es (Marzo, 2013).

Ribera, M.; Térmens, M.; Frías, A. (2009). La accesibilidad de las webs de las universidades españolas: balance 2001-2006. // Revista Española de Documentación Científica. 32:3, 66-88.

SortSite Web Site Testing Tool. http://www.powermapper. com/index.htm (Marzo, 2013).

UNE 139803:2012: Requisitos de accesibilidad para contenidos en la web. Madrid: Asociación Española de Normalización y Certificación, 2012.

Web content accessibility guidelines (WCAG 2.0) (2008). World Wide Web Consortium. http://www.w3.org/ TR/WCAG (Enero, 2013).

Enviado: 2012-04-05. Segunda versión: 2013-07-15. Aceptado: 2013-08-23. 
